\title{
Is it right to estimate inter-modular connectivity from local field potentials?
}

\author{
Xue-Mei Cui ${ }^{1,2}$, Won Sup Kim², Dong-Uk Hwang ${ }^{3}$, Seung Kee Han² \\ From 24th Annual Computational Neuroscience Meeting: CNS*2015 \\ Prague, Czech Republic. 18-23 July 2015
}

Human brains with hundreds of billions of neurons are organized in a hierarchical modular network. There have been many attempts to estimate inter-modular connectivity utilizing coherent neuronal activities of a huge number of neurons, such as the electro-encephalogram, the magneto-encephalogram, and the functional magnetic resonance imaging. Here we ask a question: Is the inter-modular connectivity estimated from the modular activities consistent with the inter-modular connectivity that could be extracted from the network connectivity of individual nodes?

To answer this question, we introduce a method of estimating the inter-modular connectivity based on the analysis of inverse phase synchronization [1,2]. For coupled phase oscillators on a hierarchical modular network shown in the Figure 1(A), the local field potential corresponding to a module is defined as the mean phase of oscillators belonging to a subset of the module.
(A)

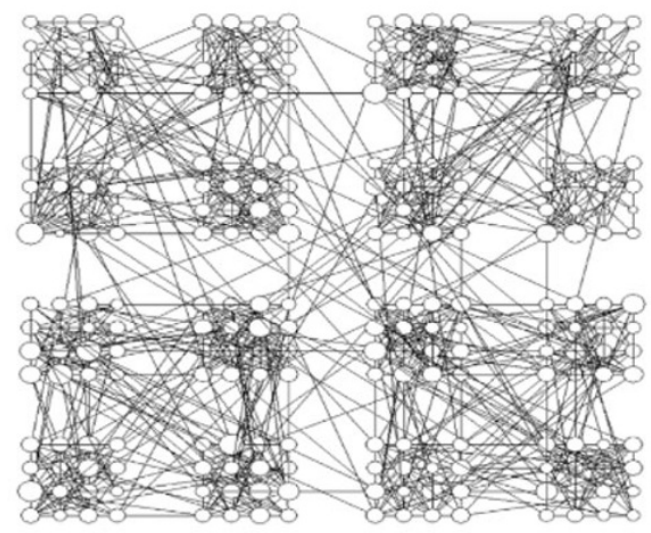

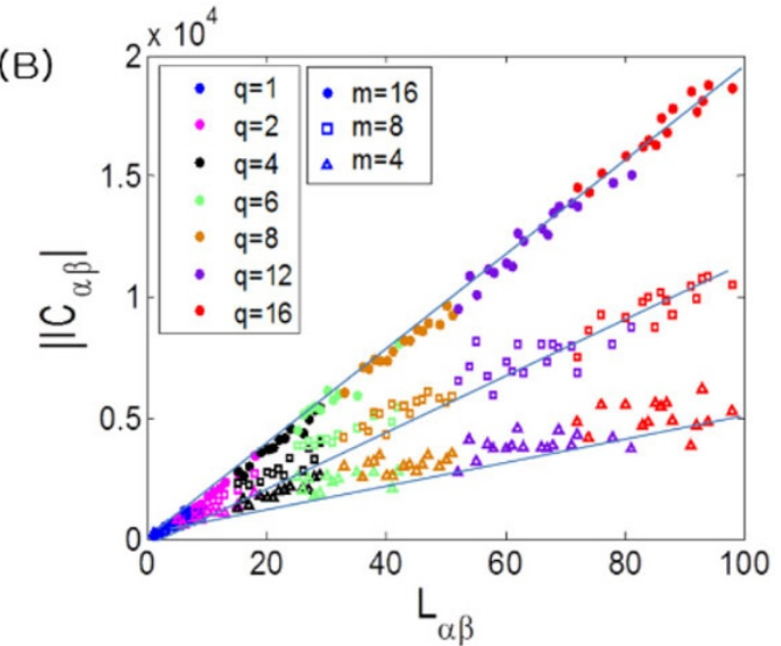

Figure 1 (A) A hierarchical modular network model 256-p-q-r, where $p$ denotes the number of links of one node with nodes of its lower-level module, $q$ links with nodes of the rest modules in its upper-level module, and $r$ links with nodes of any modules from the rest of the network. Here we present an example of $p=13, q=4$ and $r=1$. (B) Plot of the inverse phase synchronization index versus the number of links connecting two modules. The linear dependence between the inverse phase synchronization index and the number of links connecting two modules is shown when the local field potential is taken over $m$ oscillators belonging to a subset of each module.

\footnotetext{
* Correspondence: skhan@chungbuk.ac.kr

${ }^{2}$ Department of Physics, Chungbuk National University, Cheongju, Chungbuk

361-763, Republic of Korea

Full list of author information is available at the end of the article
}

(C) 2015 Cui et al. This is an Open Access article distributed under the terms of the Creative Commons Attribution License (http:// creativecommons.org/licenses/by/4.0), which permits unrestricted use, distribution, and reproduction in any medium, provided the original work is properly cited. The Creative Commons Public Domain Dedication waiver (http://creativecommons.org/publicdomain/ zero/1.0/) applies to the data made available in this article, unless otherwise stated. 
For strong coupling strength, it is shown in Figure 1 (B) that the inverse phase synchronization index grows linearly with the number of links connecting two modules. This result enables us to estimate the inter-modular connectivity in various complex systems from the inverse phase synchronization index of the mesoscopic modular activities.

\section{Authors' details}

${ }^{1}$ Normal College, Yanbian University, Yanji 133002, China. ${ }^{2}$ Department of Physics, Chungbuk National University, Cheongju, Chungbuk 361-763, Republic of Korea. ${ }^{3}$ National Institute of Mathematical Sciences, Daejon 305811, Republic of Korea.

Published: 18 December 2015

\section{References}

1. Schelter B, Winterhalder M, Dahlhaus R, Kurths J, Timmer J: Partial phase synchronization for multivariate synchronizing systems,. Phys. Rev. Lett 2006, 96:208103.

2. Kim S, Cui X-M, Yoon CN, Ta HX, Han SK: Estimating network link weights from inverse phase synchronization indices. Europhys. Lett 2011, 96:20006.

- Convenient online submission

- Thorough peer review

- No space constraints or color figure charges

- Immediate publication on acceptance

- Inclusion in PubMed, CAS, Scopus and Google Scholar

- Research which is freely available for redistribution 